\title{
Plasma lipoproteins and cellular metabolism
}

\author{
from J. S. Owen
}

Plasma lipoproteins are macromolecular complexes in which lipids are bound to a variety of polypeptides (the apoproteins) through non-covalent forces. Four main classes of lipoprotein are recognized, each defined by the density at which it floats in the ultracentrifuge chylomicrons, very-low-density lipoprotein (VLDL), low-density lipoprotein (LDL) and high-density lipoprotein (HDL). Although these classes vary widely in size, and in lipid and apoprotein composition, they appear to have a similar general structure: they are spherical particles with a hydrophobic core of cholesteryl ester and triglyceride surrounded by a $2 \mathrm{~nm}$-thick monolayer of apoprotein, cholesterol and phospholipid molecules.

Chylomicrons transport triglyceride and other fat-soluble substances from the intestine while VLDL transports endogenous triglyceride from the liver. The triglyceride contained in these particles is delivered to extrahepatic tissues, thereby providing cells with an important energy source. Although almost all cells can synthesize cholesterol, several clinical and biochemical studies suggest that most cellular cholesterol is derived from uptake and degradation of LDL. By contrast, one function of HDL may be to transport cholesterol from peripheral tissues to its principal site of catabolism and excretion in the liver.

These lipid transport processes are complex; they depend on an interchange of constituent lipids and apoproteins amongst the various lipoproteins, whether by exchange collision or by specific transfer proteins, and the activities of lipoprotein lipase, hepatic lipase and lecithin-cholesterol acyltransferase (LCAT). Other aspects of intravascular lipoprotein metabolism being investigated include the relationship between the various HDL subclasses and the mechanism by which specific HDL particles remove cellular cholesterol (Tall and Small Adv. Lipid Res. $17 ; 1,1980$ ), the origin of the lipoproteins producing cholesteryl ester deposition in the macrophage-like cells of the arterial wall (Goldstein et al. J. biol. Chem. 255; 1839, 1980, and Schechter et al. J. Lipid Res. 22; 63, 1981), and the involvement of the liver in $\mathrm{LDL}$ and $\mathrm{HDL}$ clearance (Pittman et al. Proc. natn. Acad. Sci. U.S.A. 76; 5345, 1979). In contrast, one aspect of lipoprotein metabolism has been relatively neglected: the influence of lipoproteins on cellular function by their regulation of selected cellular biochemical reactions and by their role in maintaining a normal cell membrane lipid composition.

The interaction of circulating hormones and certain non-hormonal molecules with specific cell-surface membrane receptors is an important method of regulating cellular metabolism. Plasma lipoproteins also appear to possess this regulatory capacity. The elegant work of $\mathbf{J}$. Goldstein and $\mathbf{M}$. Brown at the University of Texas, Dallas has established that cultured cells have high-affinity receptor sites which recognize the apoprotein moiety (ApoB) of LDL. Subsequent studies, principally by $R$. Mahley and colleagues at the National Heart and Blood Institute, Bethesda, have shown that ApoE-containing lipoproteins, including both HDL, (the HDL sub-class through which cholesterol may be delivered to the liver) and chylomicron remnants (the particles remaining after partial hydrolysis of core triglyceride), also bind to the same cell-surface receptor. After binding, these various lipoproteins regulate intracellular cholesterol metabolism and receptor activity, processes dependent on cellular uptake and degradation of lipoproteins.

Current evidence suggests that cells may also have additional lipoprotein receptors, specific for the apoprotein moieties and distinct from the classical 'LDL receptor', and that lipoprotein occupancy of such receptors is sufficient to modulate selected cellular functions without requiring internalization of the intact particles. These include the activity of membranebound enzymes such as adipocyte adenylate cyclase and erythrocyte $\mathrm{Mg}^{2+}-$ ATPase which are stimulated by VLDL and LDL, the enhancement of lipogenesis in fat cells by VLDL and the apparent LDL-induced reduction of prostacyclin production in endothelial cells. However, the type of regulation most extensively studied has been the potent inhibition of mitogen-induced lymphocyte proliferation by the ApoB- and ApoE-containing lipoproteins (Hui et al. J. biol. Chem. 255; 11755,1980 ). J. Harmony and associates at Indiana University have shown that several primary mitogen-induced membrane events in lymphocyte transformation, such as calcium ion and cyclic GMP accumulation and phosphatidylinositol turnover, are markedly suppressed by membranereceptor binding of these apoproteins.

The membrane lipid composition of cells may be influenced by lipoprotein lipid connposition as a consequence of the ready exchange between lipoprotein cholesterol and phospholipid (mainly phosphatidylcholine and sphingomyelin) and the corresponding lipids in cellular plasma membranes. Although cell membrane

J. S. Owen is in the Academic Department of Medicine, The Royal Free Hospital, London. transport, receptor and enzymatic functions are primarily determined by the protein constituents, there is considerable evidence that such membrane protein functions may be influenced by the properties of the fluid lipid bilayer matrix. A shift in the dynamic equilibrium between membrane and lipoprotein lipids, through abnormalities in lipoprotein lipid pattern, would therefore be expected to lead to changes in membrane lipid composition and so indirectly to membrane and cellular dysfunction. For example, the ratio of cholesterol to phospholipid is an important determinant of membrane fluidity, many membrane-bound enzymes depend on specific phospholipids for full activity, and phospholipid fatty acid composition may be a critical factor in secondary modulation of fluidity and in synthesis of prostaglandins.

Is there experimental evidence that this potential influence of plasma lipoproteins on cellular metabolism, through their effects on either membrane lipid composition or regulation of selected biochemical reactions, is physiologically important? Support has largely been obtained by study of those dyslipoproteinaemias, associated with either genetic, pathological, hormonal or dietary disturbances, in which substantial changes in apoprotein and lipid patterns have occurred. In this context, reports by $\mathrm{N}$. McIntyre and his group at the Royal Free Hospital, London on the cellular abnormalities associated with the acquired LCAT deficiency of liver disease are of interest. Lipid changes were found in erythrocytes and platelets and the abonormalities correlated with cellular dysfunction (Owen et al. J. Lipid Res. 22; 423, 1981); there was also evidence in patients with liver disease that their abnormal HDL, enriched in ApoE, could effect cellular regulation by reducing LDL uptake, by altering erythrocyte morphology and by suppressing lymphocyte proliferation. However, the majority of cells studied in such cases of abnormal lipoprotein composition have been isolated from the lipoprotein-rich environment of the plasma compartment and clearly there is a need to extend these observations to cells which have access only to the lipoproteins of the extravascular fluid. That such investigations will be fruitful is suggested by the interactions of abnormal lipoproteins with cultured cells and by the poorly understood metabolic and cellular disturbances frequently associated with those dyslipoproteinaemias, such as primary and secondary LCAT deficiency and abetalipoproteinaemia, in which marked changes in lipid and apoprotein composition occur. 